rev.relac.int.estrateg.segur.10(2):197-214,2015

\title{
LA NEUTRALIDAD ACTIVA, ENFOQUE COMPARATIVO ENTRE LA POLÍTICA EXTERIOR COLOMBIANA Y TURCA*
}

\author{
Marcela Guerrero Turbay** \\ Mauricio Jaramillo Jassir ${ }^{* * *}$
}

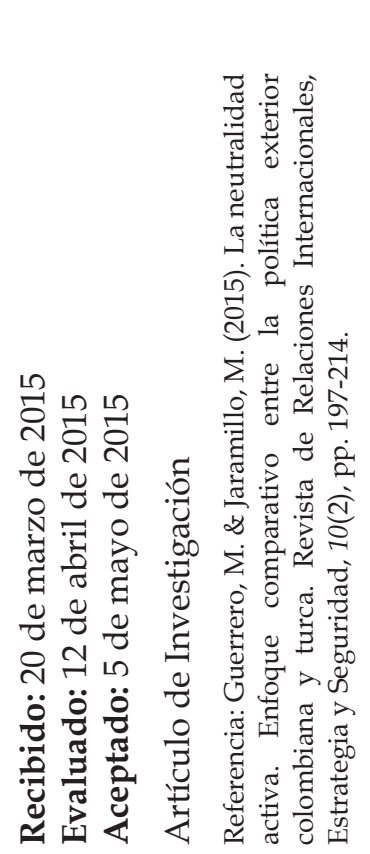

\section{RESUMEN}

En lo corrido del siglo, los gobiernos de Colombia y Turquía han emprendido una agresiva campaña en política exterior con el objeto de lograr reconocimiento internacional y visibilidad

* El artículo es el resultado del proyecto de investigación "Poder blando y diplomacia cultural de Turquía. Análisis de su proyección regional y global, y las lecciones para Colombia" escogido en la convocatoria del Fondo de Investigaciones de la Universidad del Rosario (FIUR) en 2012 y que ha venido siendo desarrollado en 2013 con el apoyo de la Embajada de Turquía en Colombia y el Comité del Bicentenario de la Alcaldía de Barranquilla.

** Internacionalista de la Universidad del Rosario. Asistente de Investigación del Centro de Estudios Políticos e Internacionales de la Universidad del Rosario y profesora auxiliar de la asignatura Política Exterior Comparada. Correo electrónico: marcela.guerrero@urosario.edu.co.

*** Internacionalista de la Universidad del Rosario. Máster en Relaciones Internacionales del Instituto de Estudios Políticos de Touluse (2005) y en Geopolítica de la Universidad París VIII (Vincennes à Saint-Denis). Aspirante a Doctor en Ciencia Política en la Universidad de Ciencias Sociales de Touluse. Profesor de Carrera e investigador en el Centro de Estudios Políticos e Internacionales de la Universidad del Rosario. Profesor titular de las asignaturas: Geopolítica, Política Exterior Comparada y Nuevas Nociones de Seguridad Internacional. Correo electrónico: mauricio.jaramilloj@urosario.edu.co. 
como actores de relevancia. Esta proyección debe entenderse en sus debidas proporciones, pues ninguno pretende convertirse en potencia regional porque carecen de atributos de poder para acceder a tal status y porque quieren evitar las responsabilidades que eso conlleva. Por ende, la opción más viable para lograr el reconocimiento como líderes o potencia emergentes, consiste en la neutralidad, acompasada de las posibilidades de mediación, y en su carácter de economías en crecimiento. El presente artículo de reflexión busca, por tanto, observar las posibilidades de Ankara y Bogotá a la luz de la neutralidad activa como elemento de atracción, y que se ha venido convirtiendo en derrotero de sus políticas exteriores.

Palabras clave: Economía Emergente, Neutralidad Activa, Política Exterior Colombiana, Política Exterior Comparada, Política Exterior Turca.

\title{
ACTIVE NEUTRALITY, A COMPARATIVE APPROACH BETWEEN COLOMBIAN AND TURKISH FOREIGN POLICY
}

\begin{abstract}
During XXI century, Colombia and Turkey have entailed an aggressive foreign policy strategy in order to gained international recognition and some importance as key actors in global arena. This projection must be understood in its real dimension, since neither pretend to became as a regional power to avoid some responsibilities from that condition. Moreover, neither Turkey nor Colombia could claim such status, since they lack some national power elements. Therefore, the most effective instrument to achieve regional and international acknowledgment is based on, neutrality as a foreign policy strategy, that could represent an eventual mediation role, and in economic growth. In consequence, this article aims to determine Ankara and Bogota possibilities to use neutrality as an attraction element, and how it is become a constitute principle of their foreign policies.
\end{abstract}

Keywords: Emerging Economy, Active Neutrality, Colombian Foreign Policy, Comparative Foreign Policy, Turkish Foreign Policy.

\section{A NEUTRALIDADE ATIVA, UM ENFOQUE COMPARATIVO ENTRE A POLÍTICA EXTERIOR COLOMBIANA E TURCA}

\section{RESUMO}

Desde o princípio deste século, os governos da Colômbia e da Turquia empreenderam uma agressiva campanha de política exterior com o objetivo de lograr reconhecimento internacional e visibilidade como atores de relevância. Essa projeção deve entender-se em suas devidas proporções, pois nenhum dos países pretende converter-se em potência regional, uma vez 
que carecem do poderio necessário para ascender a tal status e porque querem evitar as responsabilidades decorrentes dessa condição. Por essa razão, a opção mais viável para lograr o reconhecimento como líderes ou potências emergentes consiste na neutralidade, somada às possibilidades de mediação, e a seu caráter de economias em crescimento. $\mathrm{O}$ presente documento busca, portanto, observar as possibilidades de Ancara e Bogotá evocando a neutralidade ativa como elemento de atração, e que se vêm convertendo em ideal de suas políticas exteriores.

Palavras-chave: neutralidade ativa, política exterior colombiana, política exterior turca, política exterior comparada, economia emergente.

\section{INTRODUCCIÓN}

Colombia y Turquía han venido consolidando sus relaciones en los últimos años, una idea que puede constatarse por dos avances claros, el acuerdo de libre comercio y la eliminación del visado que busca que la integración no sólo tenga una dimensión económica sino que de ella participen las personas como agentes de dicho proceso. Claro está, este vínculo recreado en los últimos años no debe sobreestimarse, ya que Turquía aún dista de representar un aliado estratégico, y Colombia todavía se encuentra lejos de los intereses vitales de Ankara. No obstante, su relación es llamativa no sólo por el avance mencionado, sino porque comparten una serie de características en política exterior, que para el caso colombiano son interesantes, pues Turquía cuenta con un modelo de proyección regional que puede traducir lecciones para la política exterior de Colombia.

A pesar de ser Estados con culturas distintas, y ubicarse en latitudes alejadas una de otra, Ankara y Bogotá comparten rasgos fundamentales que sobresalen en sus acciones regionales, a saber, el hecho de estar rodeados de vecinos que los ven con desconfianza, el haber convertido sus economías en crecimiento en elementos de poder de atracción, la seguridad nacional como principio elemental de su política exterior, una alianza histórica con Estados Unidos que en el último tiempo se ha venido revaluando, y una búsqueda a través de la moderación de un liderazgo regional, que no debe confundirse con el intento por convertirse en potencia. $\mathrm{Ni}$ Colombia ni Turquía pretenden acceder a tal condición, pues no cuentan con suficientes atributos de poder como para asumir la responsabilidad que eso comporta.

En consecuencia, la hipótesis central del texto es que Ankara y Bogotá han buscado convertir su excepcionalidad en una ventaja real en política exterior. Colombia ha intentado desmarcarse del discurso nacionalista de la Nueva Izquierda Latinoamericana para atraer la inversión extranjera y consolidarse como un modelo distinto en América Latina. En un principio se pensaba que la consolidación de gobiernos de izquierda en la zona era una desventaja para Colombia, no obstante se ha buscado revertir esa idea. 
Turquía, por su parte, ha creído la neutralidad activa desde hace por lo menos medio siglo. El hecho de estar entre dos realidades geopolíticas dispares (Europa y Medio Oriente) terminó en una apuesta por sacar provecho de esa ubicación. No es cierto que Turquía sea puente o Estado tapón como comúnmente se cree, sino que se han convertido en el portador de un discurso propio, que no se puede insertar ni en la lógica de Europa Occidental, ni en la del universo musulmán del que hace parte a pesar de su laicidad.

Lo cierto es que Turquía ha alcanzado un grado tal de visibilidad que su importancia en el mundo resulta irrebatible, y su modelo de proyección tiende a convertirse en un paradigma para aquellos Estados que desde el pragmatismo, buscan influir en el escenario regional y por qué no global.

El propósito central, luego de problematizar la hipótesis consiste en sugerir que Colombia aún se encuentra lejos de capitalizar esa excepcionalidad como sí lo ha hecho Turquía. Para demostrar esta tesis el documento contiene tres partes: una primera que explora los rasgos fundamentales de la neutralidad ideológica de Turquía, especialmente desde el ascenso del Partido de la Justicia y el Desarrollo (AKP). Luego se analizará, la manera en que Colombia ha transitado de una política exterior con un fuerte acento en la defensa a la búsqueda de liderazgo regional. Finalmente, el texto aporta un conjunto de reflexiones acerca de las lecciones del modelo turco para la política exterior colombiana.

\section{TURQUÍA, UNA NEUTRALIDAD IDEOLÓGICA}

La política exterior de Turquía ha estado determinada tanto por sus condiciones internas, como por coyunturas internacionales. Por lo tanto, es posible contemplar una política exterior con un margen de cambio importante, que evoluciona de acuerdo a las realidades del sistema.

Los rasgos generales más protuberantes de su política exterior, adaptada a los diferentes contextos históricos son identificados por Deringil (1989), quien los enumera así:

a. Turquía cuenta con una ubicación geográfica excepcional, pero dependiendo del contexto puede ser ventaja o desventaja.

b. Es una ventaja porque le permite atraer a otros actores del sistema que ven en su ubicación una ventaja y de esta forma convertirse en una potencia.

c. Es una desventaja porque su ubicación lo ubica en medio de la confrontación de grandes poderes.

d. La efectividad del poder turco sólo se puede medir en términos de recursos naturales, en la medida en que el mundo es anárquico, lo que impera para el ejercicio como potencia es el poder real material. 
e. Como consecuencia de esto último, Turquía debe estar siempre preparada para la defensa militar de su territorio.

f. La negociación es el instrumento por excelencia de los poderes pequeños, por ende, de esta práctica depende la supervivencia turca.

Estas características enunciadas son visibles desde la posguerra, y se puede afirmar que han prevalecido como debates permanentes de la proyección de Turquía. Resulta interesante además resaltar que no existe un consenso claro sobre la ubicación geográfica y concretamente si es ventaja o desventaja. El hecho de tener que reflexionar sobre ello ha terminado por depurar su política exterior. En contraste con Estados que asumen como una ventaja irrefutable la condición geográfica, y que por ende no reparan en los defectos de esa ubicación. Valga decir que el caso colombiano muestra en detalle la falta de reflexión sobre la condición geográfica, ya que en Colombia se ha asumido que la posición es un activo.

Volviendo a Turquía, su política exterior se puede clasificar en cuatro períodos: "el de la República, el de la Guerra Fría, el posterior a la Guerra Fría y el de los gobiernos del Partido de la Justicia y el Desarrollo (AKP, por sus siglas en turco)" (CIDOB, 2011, p.463).

En un primer momento, es posible contemplar a una Turquía aislacionista (enfocada en la consolidación interna y en la protección de su soberanía territorial), pero un rasgo que ha trascendido desde la Segunda Guerra Mundial ha sido el de la neutralidad activa, como lo sugiere Deringil (1989). Con el triunfo de los aliados Turquía se fue alineando poco a poco con éstos, pero vale rescatar lo siguiente: en el fondo lo que más le interesaba a Ankara era la estabilidad de la región. Para ello, lo más efectivo consistía en acercarse a Estados Unidos y a Europa Occidental, y mucho menos a la Unión Soviética. A pesar de lo que se piensa, porque se considera que Turquía se alienó con occidente en términos absolutos, se convirtió en segunda receptora de la ayuda de la URSS hacia el tercer mundo después de Cuba. Eso habla del equilibrio de sus relaciones con los bloques Este y Oeste (Kuniholm, 1991).

De manera más reciente, y con el fin de la Guerra Fría buscó insertarse en el esquema integración de la Unión Europea aunque sin conseguirlo. Actualmente, y probablemente en respuesta a esa situación se le observa como protagónica y activa. Este nuevo rol Turquía en el sistema internacional se debe, de acuerdo al académico (De Cabo, 2005), a la confluencia de factores internacionales, regionales e internos.

Por un lado,

estos factores van desde el vacío de poder dejado por la Guerra del Golfo de 1991 y la guerra contra Irak en 2003, a la dinámica cambiante de la cuestión kurda y el deterioro del conflicto árabe-israelí ... Incluyen la menguante influencia que la UE tiene ahora sobre la política exterior de Turquía, así como los EE.UU. por agravar las sensibilidades de Turquía sobre la cuestión kurda en 2003-2007 (Walker, 2012, p.8). 
Por otra parte, sería imposible explicar el desarrollo actual de la política turca sin tener en cuenta el ascenso al poder en 2002 del Partido del AKP, de corte islámico y conservador, y con una voluntad de transformar las relaciones exteriores (Walker, 2012).

Ahmet Davutoğlu, académico y actual Ministro de Relaciones Exteriores, ha sido clave en este nuevo diseño de la política exterior turca. Durante los años de academia, Davutoğlu plasmó sus lineamientos en el libro "Profundidad estratégica: Posición Internacional de Turquía". Se trata de un análisis crítico de la política exterior turca, que determinó una visión amplia de la política internacional (Kösebalaban, 2011), fundamentado en elementos geopolíticos.

La tesis de Davutoğlu define que la posición geográfica y el pasado histórico dan a Turquía características ideales para ser un actor prominente en la escena internacional. Más específicamente, la doctrina de la profundidad estratégica "subraya que Turquía está dotada de forma única, tanto por su ubicación en zonas geopolíticas de influencia, en especial por su control del Bósforo, y su legado histórico como heredero del Imperio Otomano" (Walker, 2012, p.7). Así pues, "busca cambiar la posición de Turquía de la periferia de las relaciones internacionales hasta el centro, como un actor ubicado en la intersección de múltiples regiones" (Walker, 2012, p.6).

De esta manera, se establecen una serie de nuevos principios de política exterior: el equilibrio interno entre seguridad y democracia, una política de "cero problemas" con los vecinos, el desarrollo de estrechas relaciones con las regiones adyacentes, la adhesión a una política exterior multidimensional, y la ampliación de las acciones diplomáticas (Davutoğlu, 2008).

El principio de cero problemas con los vecinos es el más notorio en la política exterior turca y "el slogan que resume las expectativas de Turquía con relación a sus países vecinos" (Ministerio de Relaciones Exteriores de Turquía, s.f.). Así pues, el gobierno del AKP "pretende mantener las mejores relaciones posibles con todos sus vecinos y los actores regionales e internacionales" (Svet, 2006, p.71). Por lo tanto, busca crear o fortalecer vínculos con países cuyas relaciones eran débiles o habían sido problemáticas en el pasado.

A esto se debe agregar que la seguridad es un elemento clave para este Estado ${ }^{1}$, y este principio: "explica aún mejor el interés regional de la política turca, puesto que sólo asumiendo un papel activo en la consecución de la paz con los países vecinos, Turquía puede esperar mantener la estabilidad interna" (Casa Árabe e Instituto Internacional de Estudios Árabes y del Mundo Musulmán, 2010, p.2).

Lo anterior implicó acercamientos con los países que no fueron una prioridad en su política exterior durante el siglo XX, como aquellos del Medio Oriente, del Cáucaso y los Balcanes

1. Se debe tener en cuenta de manera específica el factor kurdo. 
Occidentales, a través de lazos económicos y diplomáticos. Por un lado, Davutoğlu ha enfatizado en "la necesidad de construir fuertes vínculos económicos con todos los Estados de la región" (Murinson, 2006, p.953). Por otra parte, "Turquía se ha reafirmado como un poder central en el Medio Oriente considerándose como un mediador natural en los conflictos internos y regionales" (Kösebalaban, 2011, p.175).

Se debe aclarar que la legitimidad del rol de mediador de Ankara en Medio Oriente se desprende de una proyección exterior basada en el concepto de soft power, es decir:

Un nuevo esquema de proyección que a su vez da cuenta de la evolución de los atributos de poder, que actualmente tienen más que ver con: a. capacidades de influenciar económicamente; b. de evidenciar coincidencias o compatibilidades culturales (especialmente con el mundo musulmán); y c. de mostrar como exportable un modelo político que ha superado pruebas. (Jaramillo \& Guerrero, 2013)

No en vano, según una encuesta del Turkish Economic and Social Foundation en 2009, Turquía se constituyó, entre la población árabe, como la nación no-árabe más admirada, con niveles de popularidad que superaban el $70 \%$.

En concordancia con lo anterior, ha diversificado sus socios económicos entablando vínculos comerciales con Bulgaria y Rumania, miembros de la Unión Europea desde 2007, así como con Ucrania, país con el que se planea firmar un tratado de libre comercio. De la misma forma, el gobierno del AKP ha desarrollado una estrategia de cooperación regional con los países del Cáucaso. Además, se ha esmerado en el fortalecimiento de sus relaciones con Azerbaiyán y Georgia. Para este último se ha seguido una política orientada a la búsqueda de una solución a los conflictos con Abjasia y Osetia del Sur (Ministerio de Relaciones Exteriores de Turquía. s.f.).

Asimismo, Siria e Irán ejemplifican el éxito de la política de Davutoğlu. En primer lugar, Siria como un caso relevador de la asertividad diplomática y económica en Medio Oriente (Kösebalaban, 2011). Aunque durante muchos años las relaciones turco-sirias estuvieron dominadas por la preocupación acerca del apoyo de Damasco al Partido de los Trabajadores de Kurdistán (PKK por sus siglas en turco), disputas sobre los recursos de los ríos Tigris y Éufrates, y los reclamos sirios sobre la provincia de Hatay (Kirişci, 2000). En la primera década del siglo XXI, se estableció un tratado de libre comercio, se eliminó el visado turístico y Turquía sirvió de interlocutor entre Israel y Siria con respecto a la disputa sobre los Altos del Golán.

Del mismo modo, vale la pena rescatar el caso iraní. Como actores con la ambición de fungir como potencias regionales de Medio Oriente, las relaciones turco-iraníes han estado marcadas por la rivalidad. No obstante, la llegada al poder de AKP permitió un acercamiento importante. Así pues, Turquía se ha dado el lujo, junto con Brasil, de mediar en las negociaciones respecto al programa nuclear iraní y se han llevado a la agenda bilateral importantes acuerdos de cooperación energética. 
Ahora bien, el acercamiento poco exitoso con Armenia, Grecia y Chipre le ha valido varias críticas a Turquía y a su política de vecindad, pero se debe tener en cuenta que el peso de la historia es determinante en las relaciones con estos países. Así pues, asuntos como el genocidio armenio, el conflicto de Nagorno Karabaj, la disputa del Egeo y la cuestión turco-chipriota son factores que no permiten un avance efectivo en el mejoramiento de relaciones.

Entre tanto, la relación con Israel pareció deteriorarse. Hace unos años, eran calificados como "aliados estratégicos", pero recientemente el declive de esta relación es patente debido, entre otros factores, al ataque de la Flotilla de la Libertad en 2010, en el que murieron nueve ciudadanos turcos.

De acuerdo al historiador Erol (2013), en una entrevista concedida a los autores, el asunto israelí

es el único aspecto de la política en la que ellos (AKP) mezclan racionalidad con emoción. $\mathrm{Al}$ igual que muchos musulmanes, apoyan la causa palestina, pero cuando se gobierna un Estado es diferente, ... están perdiendo su mejor aliado en Medio Oriente y esto va a tener repercusiones para Turquía. ¿Tienen a alguien para reemplazar a Israel? ¿Tienen algún otro aliado en el Medio Oriente, cualquier otro país estable, con el que Turquía puede cooperar?

Otras críticas que se derivan de esta política tienen que ver con el recelo con la que los países vecinos ven las aspiraciones de Turquía, por lo que se le ha acusado de promover un Neootomanismo en la región. Según Erol (2013), muchas personas en los países árabes y en los Balcanes temen un programa político expansionista turco, y reaccionan ante él de una manera nacionalista. Adicionalmente, debido al talante del AKP, hay suspicacia respecto del carácter islamista de la política exterior turca, en el que el sunismo ha cobrado importancia.

Entre otros factores de crítica, se destacan los levantamientos populares de Medio Oriente y África del Norte, que iniciaron en Túnez en 2011. Se ha planteado que Turquía ha perdido un amplio margen de maniobra en la consecución de una región estable, y que sus decisiones en política exterior lo han llevado de ser el país de los "cero problemas", al de "cero amigos".

En este sentido, el caso egipcio es revelador. Turquía apoyó las revueltas en este país, afirmando que se trataba de un cambio democrático necesario, por lo que después de la caída de Hosni Mubarak, Tayyip Receip Erdoğan, primer ministro turco, celebró la elección de Mohammed Morsi, miembro de los Hermanos Musulmanes. Una vez Morsi fue derrocado mediante el golpe militar, Ankara condenó la acción perdiendo de paso un aliado importante en la región. Ésta es una de las consecuencias, de acuerdo a Erol (2013), de tomar partido por un lado, ya que se corre el riesgo de perder cuando se escoge un segmento de una nación vecina y ésta pierde el liderazgo o el control del gobierno, como ocurrió la con la Hermandad Musulmana. 
De manera similar, la cruenta guerra civil siria parece un desafío al liderazgo turco. La apuesta de Ankara de no apoyar el régimen de Bashar Al-Assad (aunque tampoco se haya hecho manifiesto el apoyo a los rebeldes), supuso el rompimiento de los lazos económicos y diplomáticos establecidos desde 2002 y el estallido de una emergencia humanitaria, debido a la gran cantidad de sirios refugiados en Turquía. Asimismo, esta posición ha causado dificultades en la fluida relación con Irán, amigo del régimen sirio.

Así pues, Turquía se encuentra ante un panorama desolador comparado con el éxito logrado hacía pocos años. Pero si bien el gobierno turco hoy cuenta con desafíos internos ${ }^{2}$ y externos, "Turquía sigue siendo el país de mejores posibilidades en la región" (Wlaschütz, 2012, 16 de abril).

Es necesario considerar, que:

Turquía nunca pone el realismo de lado y no olvida que el enfoque de 'cero problemas' representa un objetivo y un ideal...y no es posible considerar que todos los problemas de la región...pueden resolverse en un corto período de tiempo (Ministerio de Relaciones Exteriores de Turquía, s.f.).

Además, la doctrina de Davutoğlu permitió que en la última década, Turquía se consolidara como líder en la región. Incluso, actualmente puede considerarse que el país sigue siendo un interlocutor válido en una región que normalmente es vista como conflictiva a la luz de los conflictos religiosos o políticos. Una vez más el caso sirio es ejemplar, pues Ankara es parte fundamental en la toma de decisión para la resolución de la crisis.

Aun así, el desafío turco está en la capacidad de adaptarse a las realidades internacionales y en reinventar su política como lo hizo durante el transcurso del siglo XX. La neutralidad ideológica la ha valido réditos en política exterior, y se puede decir que el mantenimiento de ese discurso también ha significado sacrificar influencia. No obstante, sus posibilidades de proyección regional e incluso global son reales.

\section{COLOMBIA ¿HACIA UNA NEUTRALIDAD ACTIVA?}

En Colombia los estudios en política exterior se han orientado en buena medida a analizar el dilema (supuesto o verdadero) entre la aquiescencia o la autonomía frente a Estados Unidos, lo

2. Turquía experimentó su propio levantamiento popular en el verano del 2013, después de que un grupo de ambientalistas protestaran en contra de la construcción de un centro comercial en el Parque Taksim de Estambul. Una protesta que empezó de forma pacífica terminó en cruentos enfrentamientos con la policía, altamente cuestionados por la comunidad internacional. Actualmente, el gobierno de Erdoğan enfrenta un grave escándalo de corrupción y se enfrenta ante el pedido de reformar el sistema judicial del país. 
que demuestra una influencia de los estudios sobre política exterior latinoamericana, sobre la producción académica nacional al respecto.

En cuanto a la literatura continental, vale la pena destacar los trabajos de Puig (1984) sobre los distintos tipos de autonomía, de Tokatlian \& Russell (2003) sobre la autonomía relacional y la aquiescencia y de Arlene Tickner (2009). En ellos se toca un tema de vital trascendencia en la política exterior colombiana y latinoamericana y que consiste en la imposibilidad de determinar su importancia como actor del sistema internacional. América Latina como parte de la periferia ha debido emprender esfuerzos concretos para ganar visibilizar con distintos efectos para los casos de Brasil, Cuba y México.

Colombia jamás fue vista como un caso aparte de proyección o no por lo menos en las dimensiones de estos tres Estados. El debate de la política exterior colombiana estuvo más bien orientado a establecer sus márgenes de acción. Esto se puede rastrear en autores que en la actualidad tienden a convertirse en los clásicos de la política exterior colombiana, como Ramírez \& Restrepo (1997), Cardona (1990), o Grekonja (1982).

Diego Cardona encuentra seis características que muestran la forma como Colombia trata de insertarse con el fin de la Guerra Fría. Estos matices son: un proceso gradual de desideologización y universalización de las relaciones internacionales; la estrecha relación entre lo interno y lo internacional; el derecho como instrumento privilegiado de política exterior; la importancia de la variable de la política económica, la utilización de la diplomacia multilateral y la limitación de instrumentos de la política exterior (Cardona, 1990).

Sin embargo, desde finales de los noventa, este panorama cambió drásticamente, ya que la internacionalización del conflicto, tendió a condicionar su proyección regional e incluso podría decirse que global. En la década de los noventa, se llegó a sugerir que Colombia disponía de un poder negativo (Carvajal \& Pardo, 2000) para utilizar en su favor, y por el protagonismo que había ganado por su guerra interna, y por su rol como país centro del narcotráfico. Paradójicamente, el país tenía un margen de acción, por los problemas que le podía generar al sistema internacional.

En este orden de ideas, la política exterior utilizó como insumo lo que ocurría dentro del conflicto colombiano, confirmando de paso, una de las características que había señalado Diego Cardona, sobre la frontera cada vez más delgada entre lo interno y lo exterior. Ahora bien, cabe señalar que en este aspecto la diplomacia tendió a concentrarse en el conflicto, pero no se puede llegar a la conclusión de que esto hubiera ocurrido en otros ámbitos de la política nacional que se hubiesen proyectado hacia afuera.

Especialmente en los dos cuatrienios de Álvaro Uribe Vélez el énfasis de la política exterior fue la defensa más que el conflicto como había ocurrido con Andrés Pastrana. El presidente Uribe buscaba convertir la política exterior en un instrumento de la seguridad. Autores como Vicente 
Torrijos, llegaron a señalar que así como durante el gobierno de Pastrana Arango se apostó por una Diplomacia por la Paz, en la administración Uribe se ejecutó una suerte Diplomacia de defensa. Torrijos llegó a asegurar que fue tal la relevancia de la defensa en los asuntos exteriores, que a su entender la entonces ministra de Defensa, Marta Lucia Ramírez se había convertido casi que en titular de la cartera de exteriores (Torrijos, 2002):

Es apenas natural que se esté formando la impresión de que la ministra de Defensa, Marta Lucía Ramírez, es también la ministra de Relaciones Exteriores. Y es apenas natural, porque el conflicto colombiano ha pasado a ser un tema transversal dominante en la agenda hemisférica y porque la conexión entre lo interno y lo externo de esta violencia es cada vez más estrecha y compleja.

Con todo ello, se podía vaticinar que en algún momento Colombia debía transitar por un aislamiento, porque la apuesta por la seguridad en materia de política exterior, implicaba alejarse en buena medida del apego irrestricto que por décadas el Estado colombiano había reivindicado como derrotero de su accionar internacional. Y dicho transición finalmente se produjo. Mientras internamente el país celebrara los triunfos militares sobre la guerrilla de las FARC, las tensiones con la comunidad regional e internacional se iban acumulando.

En una de las Operaciones más emblemáticas contra las FARC como lo fue Fénix en 2008 en la que resultó abatido "Raúl Reyes", Colombia vivió una dura sanción moral por parte de sus vecinos por haber incurrido en una violación a la soberanía de Ecuador. La protesta ecuatoriana que fue llevada a la Organización de Estados Americanos y al Grupo de Río, demostró que en términos generales ningún país apoyó a Colombia, ni siquiera tímidamente. La ausencia de una condena expresa no demuestra que no se estuviera aislado, ya que el continente nunca ha tenido los medios ni la voluntad política para sancionar a un tercer Estado, a menos de que Washington así lo requiera.

Tanto en el Consejo Extraordinario convocado por Rafael Correa en la OEA, como en la Cumbre del Grupo de Río que trató el tema, era evidente que Colombia se había alejado del consenso latinoamericano en un punto esencial para el conjunto de Estados: el respeto por los principios del Derecho Internacional.

Meses más tarde, durante la Operación Jaque en la que se rescató a un grupo de 15 secuestrados, volvió a quedar en evidencia la disparidad de la visión colombiana de su seguridad, y la percepción de actores internacionales. Una vez se hizo público el uso de emblemas de la Cruz Roja por parte de la aviación del Ejército para llevar a cabo el rescate, las críticas por parte de esta organización internacional arreciaron contra el gobierno de Uribe Vélez, por considerar esa práctica como violatoria del Derecho Internacional Humanitario (El Espectador, 2008, 16 de julio).

Todo esto fue creando un ambiente de tensión con los vecinos que no se limitó a Ecuador y a Venezuela. Es más, es probable que Estados Unidos viera con preocupación las ambiciones de 
Colombia de retar a estas naciones, ya que la retórica de la diplomacia colombiana, alimentaba y dinamizaba el discurso "anti americano y guerrerista" que tanto habían denunciado Caracas y Quito acerca de Bogotá y de Washington. El gobierno de Uribe Vélez torpemente les daba la razón. No por las operaciones militares en sí, cuyo éxito no es discutible, sino por el manejo a las reacciones de los vecinos. Cabe recordar que Colombia insistía en el apoyo de estos gobiernos en la lucha militar contra las FARC, en un intento que por obvias razones ningún latinoamericano respaldaría.

Tal vez el momento más crítico de ese aislamiento se vivió en 2009 durante la Cumbre de la Unión de Naciones Suramericanas (Unasur) en Bariloche, Argentina. Allí la mayoría de los Estados se pronunciaron en contra de la injerencia de Estados Unidos, y las acusaciones del presidente colombiano contra sus homólogos de Ecuador y Venezuela, fueron leídas como la confirmación de la voluntad colombiana de entrar en un aislamiento y por ende, abandono por su región natural.

Por esa época y por las declaraciones imprecisas del comandante de las Fuerzas Militares, General Freddy Padilla de León se asumió que por un acuerdo militar, Estados Unidos instalaría siete bases en Colombia. Inmediatamente se dio una respuesta enérgica de los vecinos que consideraban esa posibilidad como una clara intromisión de Washington en la política latinoamericana. La declaración final de dicha Cumbre hablaba sobre la importancia de evitar la injerencia de terceros. A este respecto, Cristina Fernández expresaba de manera tajante el sentimiento de la mayoría en Unasur (2009, p.12):

Hemos fijado una doctrina de la UNASUR sobre la presencia de tropas extraregionales en una nación integrante del organismo. No se puede tener un doble estándar. Se ha determinado un principio para cualquier país donde haya tropas extraregión. No importa de qué país se trate. He aclarado que no abordamos solo una cuestión fáctica sino fijando doctrina.

No obstante, con la llegada de Juan Manuel Santos se produjo un giro en la política exterior, y ésta pasó de ser un instrumento de la política de defensa, a convertirse en una prioridad para el gobierno, sin que en ello tuviera una incidencia mayor el Ministerio de la Defensa Nacional. En esa administración, se pretendió que las funciones de las carteras de defensa y de relaciones exteriores estuviesen suficientemente diferenciadas y evitar así semejante disparidad entre la visión interna sobre la seguridad colombiana y la percepción de la región sobre Colombia.

En los primeros años se pudo ver un alivio inmediato en las relaciones con los vecinos, especialmente con Ecuador y Venezuela, las dos relaciones que más habían sufrido por causa del vínculo entre la seguridad y la diplomacia. Luego vino una agresiva y dinámica política exterior para mostrar a Colombia como un ejemplo de éxito, tratando de evitar los errores cometidos por el expresidente Uribe. El cálculo del gobierno fue simple, la administración previa había cometido tres yerros mayores en cuanto a política exterior se refiere, y por ende, remediando esta situación Colombia podría ser retornar al esquema regional de integración. 
Los errores de la administración Uribe fueron los siguientes: en primer lugar, el gobierno había convertido a organismos defensores de derechos humanos, incluidos los del sistema de Naciones Unidas en enemigos del discurso sobre seguridad. Esto hacía pensar que la consolidación del Estado iba en detrimento del respeto irrestricto por los derechos humanos.

En segundo lugar, al exigir que los vecinos calificaran a las FARC como terroristas, Colombia había invertido esfuerzos de manera inútil y había desgastado su servicio exterior en un objetivo de imposible alcance. Durante por lo menos seis años se pidió que los latinoamericanos relativizaran el principio de no injerencia. Como resulta apenas obvio, se trata de un objetivo inalcanzable, especialmente para aquellos que circundan al país como Brasil, Ecuador, Perú y Venezuela.

Y en tercer lugar, el gobierno anterior jamás pareció entender la diferencia en la sensibilidad entre Estados Unidos y Europa frente al conflicto. La visión de Washington tiene raíces muy distintas de aquella europea. Mientras Estados Unidos entiende la necesidad de vencer militarmente a las FARC, porque libra su batalla contra el terrorismo en Medio Oriente y Asia Central, y además, se opone a cualquier posibilidad de un gobierno de corte marxista o leninista, Europa tiende a privilegiar la cautela. Bruselas enfatiza la necesidad de observar y entender el conflicto, no desde la óptica de la defensa (las FARC como amenaza), sino desde un punto de vista estructural, tratando de hallar las "causas objetivas de la violencia". Esto se pudo observar en el retiro del apoyo del Parlamento Europeo al Plan Colombia. En su declaración al respecto se puede identificar una diferencia fundamental con la versión estadounidense del conflicto (Parlamento Europeo, 2001):

[La Unión Europea] hacer constar que el Plan Colombia comporta aspectos que son contrarios a las estrategias de cooperación y a los proyectos en los cuales la Unión se ha comprometido, y que ponen en riesgo sus programas de cooperación, y se declara particularmente preocupada frente a la situación actual en la región de Putumayo ${ }^{3}$

Así se conformó una estrategia de política exterior, buscando desmarcase de estos errores y con una diferencia substancial: el uso de instrumentos diplomáticos, no tanto para aislar a las FARC y justificar el discurso de la seguridad, sino en aras de legitimar la política de paz. Con esto el gobierno separó el tema de seguridad de la política exterior, pero vinculó a esta última con la paz. Una estrategia que la ha dado resultados.

La llegada de Santos ha estado acompañada de varios frentes en la política exterior y ha buscado convertir su condición de excepcionalidad en términos ideológicos, por ser una nación

3. Traducción libre de los autores, en el texto original aparece así: fait observer que le plan Colombie comporte des aspects qui sont contraires aux stratégies de coopération et aux projets dans lesquels l'Union s'est déjà engagée, et qui mettent en péril ses programmes de coopération, et se déclare particulièrement inquiet devant la situation actuelle dans la région du Putumayo. 
tradicionalmente conservadora rodeada de gobiernos de izquierda en una ventaja. Algo que el gobierno anterior veía como una amenaza porque entendía que había un vecindario hostil.

Como consecuencia de este giro, Colombia pretende transformarse en un líder regional apoyándose en dos condiciones; en la posibilidad de convertirse en un paradigma de Estado que puede revertir una situación desfavorable y erigirse en modelo, y en capitalizar la oportunidad para que su moderación ideológica le permita estar ajena de la radicalización que afecta la imagen internacional de los Estados.

\section{LECCIONES PARA COLOMBIA}

La idea de Colombia como líder regional coincide con un momento de esplendor de la proyección de Turquía. La autonomía que mostró frente al tema nuclear iraní, su papel en la reconstrucción de Irak, su postura frente al conflicto entre Israel y los palestinos, y la distancia asumida frente a la Unión Europea terminaron por favorecer su imagen. En este escenario, la crisis económica de algunos países europeos, como España, Italia, Grecia y Chipre mostró que mientras sus vecinos occidentales atravesaban por una de las peores crisis, Turquía se reafirmaba como una economía emergente. Entretanto, Colombia ha impulsado esquemas como la Alianza del Pacífico, y su eventual entrada a la Organización para la Cooperación y del Desarrollo Económico (OCDE) debían confirmar una vocación internacionalista y multilateral retomada por la administración Santos.

Así como Turquía se ha constituido como modelo para el mundo árabe y musulmán, para Colombia puede serlo desde el punto de vista de una potencia regional y economía emergente.

El actual gobierno ha promovido a Colombia como "país puente", "bisagra" o "articulador". De hecho, "en el discurso inaugural de la Cumbre [de las Américas] por parte del presidente Juan Manuel Santos el 14 de abril, se mencionó 12 veces el concepto de 'puente' como el paradigma que propone Colombia para conducir las relaciones entre los países de las Américas" (Carvajal, 2012, p.5). De igual manera lo manifestó Luis Alberto Moreno, presidente del BID, en entrevista con El Espectador, afirmando que "Colombia es una bisagra entre el Norte y el Sur" (Carvajal, 2012, p.4).

De esta misma forma Colombia percibe el rol de Turquía en el sistema internacional. Empero, esta es una idea que debe revisarse, no sólo respecto al rol de Ankara en el mundo, sino desde el punto de vista de la estrategia que desde Bogotá se ha planeado para proyectarse en el exterior.

Con esta consideración, el ministro Davutoğlu ha rechazado enérgicamente "la percepción de Turquía como puente entre Islam y Occidente, ya que relegaría a Turquía a un instrumento para la promoción de los intereses estratégicos de otros países" (Grigoriadis, 2010, p.4). Hoy 
se habla de un Estado con vocación de potencia central, con una política independiente y proactiva, que persigue el interés nacional y no el de terceros.

Así pues, se debe ver a una Turquía multidimensional, es decir, un país medio oriental, balcánico, caucásico, centro asiático, mediterráneo, y un país del Golfo y del Mar Negro, que simultáneamente puede ejercer influencia en todas estas regiones y reivindicar un rol global estratégico (Grigoriadis, 2010).

Colombia, en ese sentido, debe concretar la idea de convertirse en puente, bisagra o punto de conexión entre dos mundos, ya que hasta el momento ha sido incapaz de aprovechar en su real dimensión la salida a dos océanos. Aunque todos los gobiernos sugieren la importancia de la posición geográfica, la disparidad entre la proyección hacia el Atlántico profusa, con la precariedad de la inserción en el océano Pacífico desnuda las limitaciones estructurales para reivindicar con legitimidad, una posición privilegiada que redunde en posibilidades y en acciones concretas en el plano de la diplomacia.

En términos generales, tres lecciones se pueden extraer del modelo turco. En primer lugar, la neutralidad activa en el caso de Turquía ha comenzado con un debate acerca de la posición geográfica, sin asumir que ésta necesariamente signifique una ventaja. Desde ese punto es posible entender una reflexión constante para incidir de manera significativa en su entorno. En Colombia se contempla poco la posibilidad de discutir las vulnerabilidades que se desprenden del acceso de dos océanos. Una vez se identifiquen es más probable entender el papel en la región.

En segundo lugar, al acercamiento de Ankara con sus vecinos ha sido una constante en los últimos años. Su acercamiento a los palestinos, a la población kurda en Irak, a la flexibilización en el esquema de negociación con Irán así lo demuestran. Colombia, por su parte, ha tratado a sus vecinos de manera inconstante pasando de la retórica de la confrontación a la del diálogo, y sin pensar en la Región Andina y en América del Sur en el largo plazo. Si Colombia desea como Turquía, desempeñar un papel a partir de su neutralidad, deberá empezar por comprender mejor a sus vecinos. En ese sentido, urgen centros de estudio para se promocione el conocimiento de los países de la zona.

Finalmente, Turquía otorga grandes lecciones en la forma en que reivindica su autonomía y dignidad frente a Europa y a Estados Unidos. La inserción de Turquía en la esfera Occidental, no ha significado una renuncia a su entorno natural, y aunque no ha encontrado un equilibrio perfecto en sus relaciones entre Occidente y Oriente, ha sido capaz de mostrar un modelo de política exterior que la ha convertido en protagonista. Colombia todavía no entiende que las relaciones con Estados Unidos no deben acarrear un abandono.

Una vez en práctica, algunas de estas reflexiones es más probable que Colombia se dote de una neutralidad activa que le permita incidir en el devenir del continente. De lo contrario seguirá atrapado en una retórica marcada por las contradicciones. 


\section{REFERENCIAS}

Cancillería (s.f.) (2013, 5 de diciembre). Ministerio de Relaciones Exteriores: Cancillería. Recuperado el, de http://www.cancilleria.gov.co/ministry/policy

Cardona, D. (1990). Características de la política exterior colombiana. Colombia Internacional, 3-9.

Carvajal, L. (mayo de 2012). Centro de Pensamiento Estratégico - Ministerio de Relaciones Exteriores de Colombia. Recuperado de http://www.cancilleria.gov.co/sites/default/ files/pensamiento_estrategico/documentos_sobre_region/b.Colombia\%20como\%20 Pa\%EDs\%20Puente\%20en\%20Pol\%EDtica\%20Exterior\%20Retos\%20y\%20Fortalezas\%20 -\%20Mayo\%202012\%20-\%20Leonardo\%20CARVAJAL.pdf

Carvajal, L., \& Pardo, R. (2003). La internacionalización del conflicto doméstico y procesos de paz. En Cardona, D. (Ed.), Desafíos y prioridades de la política exterior colombiana (pp. 181-236). Bogotá: Fescol.

Casa Árabe e Instituto Internacional de Estudios Árabes y del Mundo Musulmán. (2010). Recuperado de http://www.casaarabe.es/publicacions/index/atalaya-sociopolitica/pag/2

Barcelona Centre for International Affairs, CIDOB. (2011). Anuario internacional CIDOB 2011. Turquía, perfil del país. Recuperado de http://www.cidob.org/es/publicaciones/anuarios/ anuario_internacional_cidob/anuario_internacional_cidob_2011_turquia_perfil_de_pais

Davutoğlu, A. (2008). Turkey's Foreign Policy Vision: An Assesment of 2007. Insight Turkey, 77-96.

De Cabo, I. (2005). El Imperio Otomano. En De Cabo, I. Turquía, Grecia y Chipre: Historia del Mediterráneo Oriental (págs. 17-39). Barcelona: Publicacions I Edicions de la Universitat de Barcelona.

Deringil, S. (1989). Turkish foreign policy during Second World War. Cambridge: Cambridge University Press.

Gobierno reconoce que utilizó el emblema de la Cruz Roja en la Operación Jaque (2008, 16 de julio). El Espectador. Recuperado de http://www.elespectador.com/noticias/judicial/ articulo-gobierno-reconoce-utilizo-emblema-de-cruz-roja-operacion-jaque

Erol, E. (2013, 5 de noviembre). Identidad y política exterior turca. (M. Guerrero Turbay, Entrevistadora). 
Grekonja, G. (1982). Colombia, política exterior. Bogotá: Universidad de los Andes.

Grigoriadis, I. (2010, diciembre). Hellenic Foundation for European \& Foreign Policy. Recuperado de http://www.eliamep.gr/en/all-publications/working-papers/the-davutoglu-doctrineand-turkish-foreign-policy/

Jaramillo, M., \& Guerrero, M. (2013). El poder blando y la diplomacia cultural de Turquía: Análisis de los factores históricos y regionales. Revista de Relaciones Internacionales, Estrategia y Seguridad, 61-84.

Kirişci, K. (2000). Turkey and the Muslim Middle East. En A. Makovsky, \& S. Sayarı, Turkey's New World: Changing Dynamics in Turkish Foreign Policy (pp. 39-58). Washington: The Washington Institute for Near East Policy.

Kösebalaban, H. (2011). An emerging power in the age of globalization. En H. Kösebalaban, Turkish Foreign Policy: Islam, Nationalism, and Globalization (pp. 145-186). Nueva York: Palgrave Macmillan .

Kuniholm, B. (1991). Turkey and the West. Foreign Affairs, 34-48.

Los presidentes de la Unasur acordaron en Bariloche "la no injerencia en asuntos internos de los pueblos" (2009, 28 de agosto). Página 12. Recuperado de http://www.pagina12.com. ar/diario/ultimas/20-130772-2009-08-28.html

Murinson, A. (2006). The Strategic Depth Doctrine of Turkish Foreign Policy. Middle Eastern Studies, 945-964.

Parlamento Europeo. (2001). Résolution du Parlement européen sur le plan Colombie et le soutien au processus de paix en Colombie. Bruselas: Unión Europea.

Policy of zero problems with our neighbors, (s.f.). Ministry of Foreign Affairs of the Republic of Turkey. Recuperado de http://www.mfa.gov.tr/policy-of-zero-problems-with-ourneighbors.en.mfa

Puig, J. (1984). Introducción. En Puig, J. (Comp.), América Latina: políticas exteriores comparadas . Buenos Aires: Grupo Editorial Latinoamericano.

Ramírez, S., \& Restrepo, L. (1997). Colombia entre la inserción y el aislamiento. La política exterior colombiana en la años noventa. Bogotá: Universidad Nacional de Colombia.

Svet, O. (2006). Turkey's "Zero Problem" Foreign Policy: An Untenable Balancing Act. Insights, 71-78. 
Tickner, A. (2009). Latin America : still dependent after all these years? En O. Waever, \& A. Tickner, Global Scholarship in International Relations (pp. 32-51). Oxon: Routledge.

Tokatlian, J., \& Russell, R. (2003). De la autonomía antagónica a la autonomía relacional: una mirada teórica desde el Cono Sur . Perfiles Latinoamericanos, Facultad Latinoamericana de Ciencias Sociales, 159-194.

Torrijos, V. (2002, 27 de diciembre). Diplomacia de Defensa. El Tiempo.

Walker, J. (4 de mayo de 2012). LSE Research Online. Recuperado el 15 de enero de 2014, de http://eprints.Ise.ac.uk/43495/

Wlaschütz, C. (2012, 16 de abril). Turquía: los desafíos de un nuevo poder regional. Semana. 